\title{
Development of Quantitative Standards for Atom Probe Reconstruction Parameters for Analysis of Interfacial Chemistry
}

\author{
S.R. Broderick, S. Dumpala, S.E. Young, K. Kaluskar, S. Srinivasan, and K. Rajan \\ Department of Materials Science and Engineering and Institute for Combinatorial Discovery, Iowa State \\ University, 2220 Hoover Hall, Iowa State University, Ames, USA.
}

The present work is aimed at developing a standard for defining reconstruction parameters for optimal voxel and chemical thresholds. We develop quantitative techniques for the detection of sharp chemical interfaces from the APT outputs of 3D point cloud and 3D chemical interfaces of continuous geometry. The difficulty lies in mapping discrete data to a continuous output while minimizing the loss in chemical information. In the present work, we develop a novel approach, based on the principles of computational homology, to map the discrete 3D point cloud atomic data to the topology of a continuous chemical interface. The computational homology framework developed can be applied to APT data to get meaningful results and quantify sensitivity of the output on the data reconstruction parameters. To incorporate evaporation physics with chemistry and structure and to provide physical definition of uncertainty in spatially defining phases,

Identifying nanoscale chemical features from atom probe tomography (APT) data routinely involves adjustment of voxel size as an input parameter, through visual supervision, making the final outcome user dependent, reliant on heuristic knowledge and potentially prone to error. This work utilizes Kernel density estimators to select an optimal voxel size in an unsupervised manner to perform feature selection, in particular targeting resolution of interfacial features and chemistries. The capability of this approach is demonstrated through analysis of the $\gamma / \gamma^{\prime}$ interface in a Ni-Al-Cr superalloy.

Further, feature extraction from APT data is usually performed by repeatedly delineating iso-concentration surfaces of a chemical component of the sample material at different values of concentration threshold, until the user visually determines a satisfactory result in line with prior knowledge. However, this approach allows for important features, buried within the sample, to be visually obscured by the high density and volume ( 107 atoms) of APT data. This work provides a data driven methodology, free of user defined boundaries, classifying different phases such as precipitates by mapping the topology of the APT dataset using a concept from algebraic topology termed persistent simplicial homology. The demonstration of interfacial analyses provides a clear quantitative logic for defining reconstruction parameters of voxel size and chemical thresholds, and therefore provides a standard for analyses of interfaces [1].

\section{References:}

[1] The authors acknowledge the support from Air Force Office of Scientific Research grants: FA955010-1-0256, FA9550-11-1-0158 and FA9550-12-1-0456; and NSF grants: ARI Program CMMI-09-389018 and PHY CDI-09-41576. 

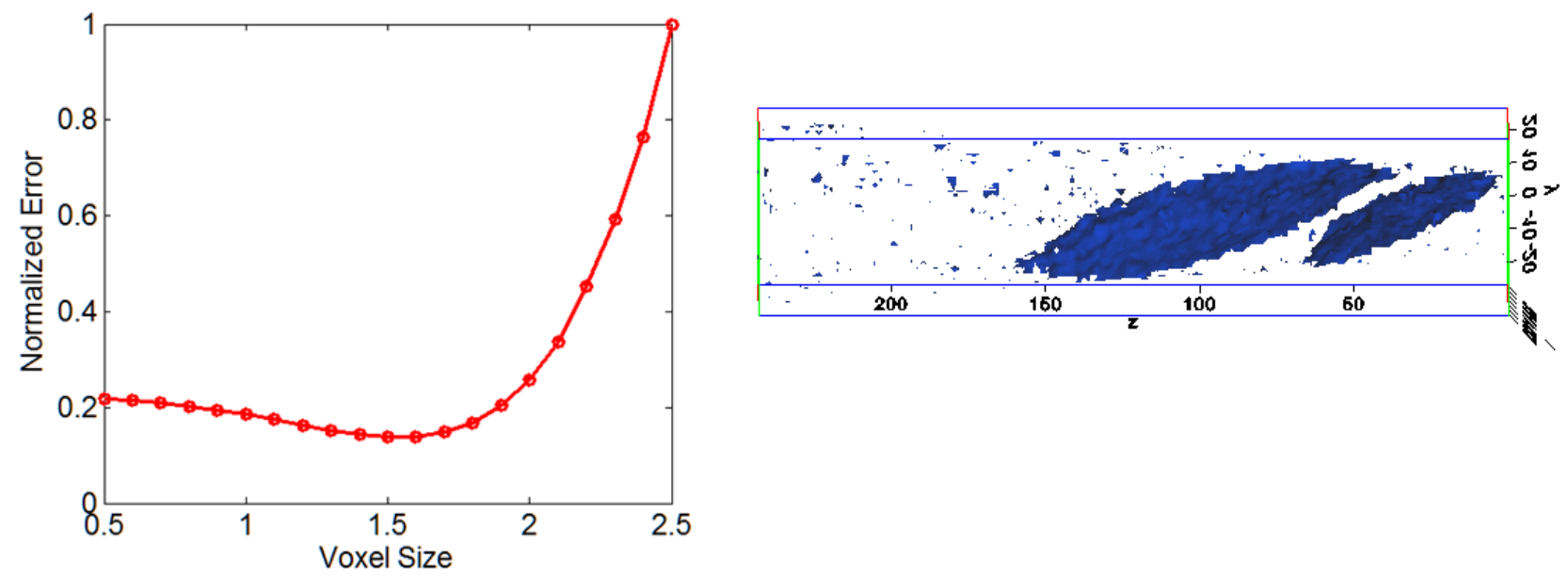

Figure 1. Definition of optimal voxel sizes for standardized definition of Ni-Al-Cr interface. (Left) Dependence of the normalized mean integrated square error (MISE) on the voxel size. The error is minimum for a voxel edge length of $1.6 \mathrm{~nm}$. (b) 3D mapping of atom positions based on voxel definitions. The effect of voxel edge length on capturing the precise interface between the $\gamma / \gamma$ ' region of the Ni-AlCr sample is captured through this analysis.
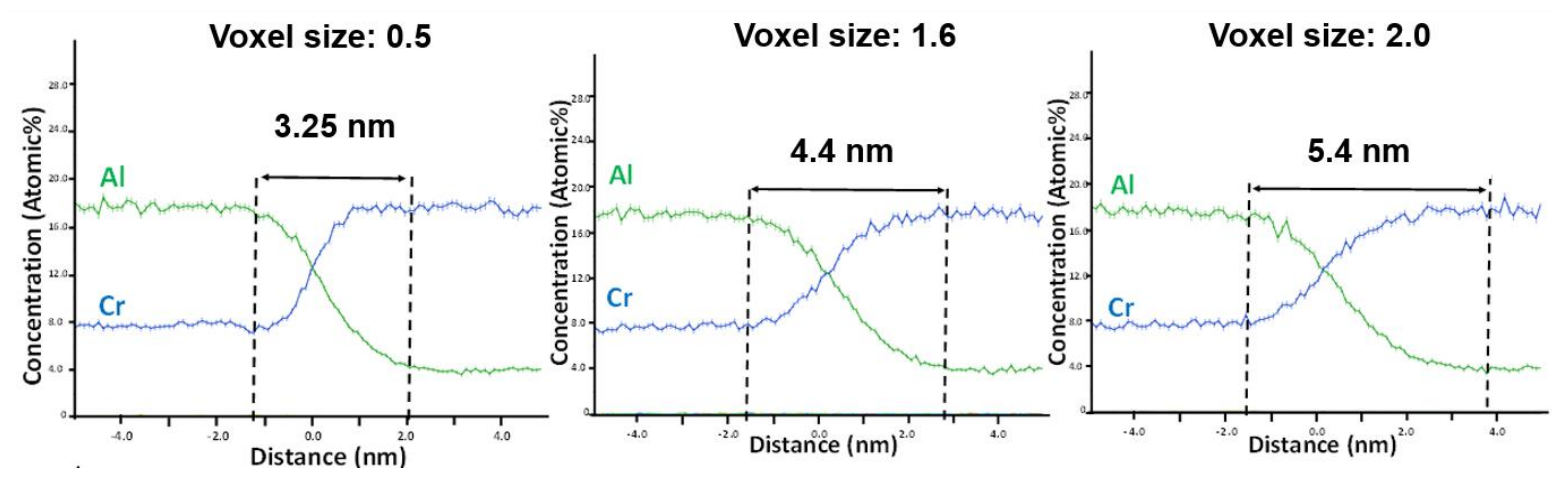

Figure 2. Concentration profiles for $\mathrm{Al}$ and $\mathrm{Cr}$ and variations in the interfacial width for different voxel sizes. From our approach, we measure the interfacial width $(4.4 \mathrm{~nm})$ while removing the inherent bias in the process. This further emphasizes the benefit of the approach, as the resulting changes in the concentration profiles are subtle, as compared with the more obvious variations in the 3D spatial mapping. We therefore through this approach capture free of bias the chemistry of the interface as measured through the profile which reflects a macroscopic measure of the phase chemistry, while the atom mapping demonstrates segregation of $\mathrm{Cr}$ to the interface which otherwise would not be identified. 